\title{
ON EXPONENTIALLY CLOSED FIELDS ${ }^{1}$
}

NORMAN L. ALLING

It is well known [4] that the non-Archimedean residue class fields $K$ of the ring of continuous real valued functions on a space are realclosed and $\eta_{1}$-sets. It does not appear to be known that the exponential function in the reals induces an exponential function in $K$ (definitions to follow); thus $K$ is exponentially closed. The property of being exponentially closed is a new invariant which will be applied to totally ordered fields in this paper.

A totally ordered field $K$ will be called exponentially closed if (i) there exists an order preserving isomorphism $f$ of the additive group of $K$ onto $K^{+}$, the multiplicative group of positive elements of $K$, and (ii) there exists a positive integer $n$ such that $1+1 / n<f(1)<n$; such an isomorphism will be called an exponential function in $K$.

In $\$ 0$ Archimedean exponentially closed fields will be considered, the rest of the paper being devoted to the non-Archimedean case. In $\$ 1$ some necessary conditions for a non-Archimedean field to be exponentially closed will be given, followed in $\$ 2$ by some examples. In $\$ 3$ a set of sufficient conditions will be given, followed by an example.

A totally ordered field $K$ will be called root-closed if $K^{+}$is divisible. Clearly exponentially closed fields and real-closed fields are rootclosed.

0. An Archimedean totally ordered field is isomorphic to a unique subfield of the reals. Let $K$ be an exponentially closed subfield of the reals and let $f$ be an exponential function in $K$. If $a=f(1)$ then $f(x)=a^{x}$ for all $x \in K$. Conversely, if $a \in K, a>1$, and if $g(x)$ is defined to be $a^{x}$ for all $x \in K$, then $g$ is an exponential function in $K$. Thus, any subfield of the reals is contained in a unique exponentially closed subfield of the reals, both having the same cardinality. The field of real algebraic numbers is, by definition, real-closed. However $2^{2^{1 / 2}}$ is not in it, hence it is not exponentially closed. It is not known to the author if exponentially closed fields need be real-closed.

1. Necessary conditions. Let $K$ be a non-Archimedean field. It is well known [3] that one can associate with $K$ a totally ordered group

Presented to the Society, April 14, 1961; received by the editors April 17, 1961 and, in revised form, July 28, 1961.

1 This research was supported by a grant from the Office of Naval Research under Contract No. Nonr-1100 (12). 
$G$ and a homomorphism $V$ of the multiplicative group of $K$ onto $G$ satisfying the following conditions: (1) $V$ is order preserving on $\mathrm{K}^{+}$, (2) $V(a \pm b) \leqq \max (V(a), V(b))(V(0)$ being the symbol $-\infty$ treated in the usual way), and (3) $V(a)=V(b)$ if and only if there exists a positive integer $n$ such that $|a| \leqq n|b|$ and $|b| \leqq n|a|$. The mapping $V$ will be called a natural valuation on $K$; clearly any two such mappings are essentially identical. The valuation ring of $V$ is $O=\{a \in K: V(a) \leqq 0\}$ and its maximal ideal $P=\{a \in K: V(a)<0\}$. Clearly the residue class field of $K, O / P=k$, is an Archimedean field.

Assume, in addition, that $K$ is exponentially closed and that $f$ is an exponential function in $K$.

LEMMA 1.1. The restriction of $f$ to $O$ maps $O$ onto the group of positive units of $O$. Further, $a \in P$ if and only if $f(a)-1 \in P$.

Proof. Since $f(1)<n, f$ maps $O$ into the positive units of $O$. Let $a$ be a positive unit of $O$. There exists $m \in N$, the set of positive integers, such that $1 / m<a<m$. Let $b=f^{-1}(a)$. It suffices to show that $b \in O$. There exists $i \in N$ such that $(1+1 / n)^{i}>m$. Thus $f(i)=f(1)^{i}$ $>(1+1 / n)^{i}>m>f(b)$, and $i>b$. Since $1+1 / n<f(1), f(-1)<n /(n+1)$. Since $n /(n+1)<1$ there exists $t \in N$ such that $(n /(n+1))^{t}<1 / m$. Thus $f(-t)=f(-1)^{t}<(n /(n+1))^{t}<1 / m<f(b)$ and $-t<b$, proving that $b \in O$ and hence the first assertion is proved.

Let $h$ be the canonical homomorphism of $O$ on to $k$. Clearly $r=h f$ is an order preserving homomorphism of $O$ onto the multiplicative group of positive units of $k$. Clearly $a \in P$ if and only if $-1<m a<1$ for all integers $m$. By condition (ii), $1+1 / n \leqq r(1) \leqq n$ and $1 / n \leqq r(-1)$ $\leqq n /(n+1)$. Hence $a$ is in $P$ if and only if $1 / n \leqq(r(a))^{m} \leqq n$ for all integers $m$ : i.e., $r(a)=1$ or equivalently $f(a)-1 \in P$, proving the lemma.

The following theorem is an immediate consequence of this lemma.

THEOREM 1.2. The residue class field of a non-Archimedean exponentially closed field is an Archimedean exponentially closed field.

The restriction of $V$ to $K^{+}$is an order preserving homomorphism onto $G$ whose kernel is the group of positive units of $O$; thus $V f$ is an order preserving homomorphism of the additive group of $K$ onto $G$ whose kernel is $O$, proving the following theorem.

TheOREM 1.3. If $K$ is a non-Archimedean exponentially closed field whose valuation ring is $O$ and whose value group is $G$ then there exists an order preserving group isomorphism that sends $K / O$ onto $G$.

It is well known [3] that given a totally ordered Abelian group $G$ 
there exists a mapping $W$ of $G$ onto a totally ordered set that has all the properties of $V$, except that of being a homomorphism. Such a mapping, characterized by these properties, will be called a natural valuation on $G$. Let $G^{+}$be the set of positive elements of $G$. Then $S=W\left(G^{+}\right)$will be called the value set of $G$. For $s \in S$ let $G$, $=\{g \in G: W(g) \leqq s\} /\{g \in G: W(g)<s\}$. Clearly $G_{s}$, which will be referred to as the factor of $G$ associated with $s$, is an Archimedean group.

Corollary 1.4. Assume that $K$ is a non-Archimedean exponentially closed field. Let $G$ be the value group of $K$ and $k$ the residue class field of $K$. Then $G^{+}$is isomorphic as an ordered set to $W\left(G^{+}\right)$and the factors of $G$ are isomorphic to $k$.

Proof. By Theorem 1.3, $K / O$ and $G$ are isomorphic; thus they have isomorphic value sets. The value set of $K / O$ under the natural valuation induced by $V$ is $G^{+}$, proving the first assertion. Let $g \in G^{+}$. The factor of $K / O$ associated with $g$ is isomorphic to the factor $K_{g}$ of $K$ associated with $g$. Let $a \in K$ such that $V(a)=g$. Then $K_{o}=O a / P a$, which is isomorphic to $O / P=k$, proving the corollary.

2. Examples. Under pointwise operations, the set $C(X)$ of all continuous functions from a completely regular Hausdorff space into the reals is a lattice-ordered ring. If $a \in C(X)$ then $e^{a} \in C(X)$; further, $a$ and $e^{a}-1$ have the same zeros and hence [4] belong to the same maximal ideals. Let $K$ be a non-Archimedean residue class field of $C(X)[4]$ and let $h$ be the associated canonical homomorphism. For $a^{\prime} \in K$ let $a \in h^{-1}\left(a^{\prime}\right)$, and let $f\left(a^{\prime}\right)=h\left(e^{a}\right)$. Since $a^{\prime}=0$ if and only if $h\left(e^{a}-1\right)=0, f$ is a well defined isomorphism of $K$ into $K^{+}$. Since $h$ and $a \rightarrow e^{a}$ are order preserving, so then is $f$. For $a^{\prime} \geqq 1$ we may choose $a \geqq 1$. Let $b=\log a$ and let $b^{\prime}=h(b)$. Clearly $f\left(b^{\prime}\right)=a^{\prime}$. For $0<a^{\prime}<1$ we may apply the argument above to $1 / a^{\prime}$; thus $K$ is exponentially closed. $^{2}$

It is well known [4] that such fields are real-closed, have the reals as their residue class field and are $\eta_{1}$-sets in the sense of the following definition. Let $\alpha$ be an ordinal number and let $T$ be a totally ordered set. $T$ is called an $\eta_{\alpha}$-set if, given subsets $A$ and $B$ of $T$ of power less than $\aleph_{\alpha}$ such that $A<B$, then there exists $t \in T$ such that $A<\{t\}<B$.

It has been shown [2] that if $\alpha>0, \boldsymbol{N}_{\alpha}$ is a regular cardinal number, and $\sum_{\delta<\alpha} 2 \aleph_{\delta} \leqq \aleph_{\alpha}$, then a real-closed field exists that is an $\eta_{\alpha}$-set of power $\aleph_{\alpha}$. Let $K$ be such a field. Clearly $K$ is non-Archimedean. Let $f_{0}(n)=2^{n}$ for all integers $n$. Both the additive group of $K$ and the

2 According to Henriksen, this argument can be used to show that the residue class fields of uniformly closed phi-algebras are exponentially closed. 
multiplicative group of positive elements of $K$ are totally ordered Abelian divisible groups that are $\eta_{\alpha}$-sets of power $\aleph_{\alpha}$. Thus by Theorem $\mathrm{B}[1] f_{0}$ extends to an exponential function in $K$, proving that $K$ is exponentially closed.

Let $k$ be an Archimedean field and let $T$ be a nonempty totally ordered set. For $a \in k^{T}$ let $s(a)=\{t \in T: a(t) \neq 0\}$. A subset of $T$ is called anti-wellordered if every nonempty subset of it has a greatest element. Let $k\{T\}$ be defined to be $\left\{a \in k^{T}: s(a)\right.$ is anti-wellordered $\}$. Clearly $k\{T\}$ is an Abelian group under pointwise addition. For $a \in k\{T\}, a \neq 0$, let $d(a)$ be the greatest element in $s(a)$. Define $a>0$ if $a(d(a))>0$; then $k\{T\}$ is a totally ordered group, $d$ is a natural valuation and $T$ is its value set.

For an ordinal number $\alpha$ let $k\{T\}_{\alpha}=\{a \in k\{T\}$ : the cardinal number of $s(a)$ is less than $\left.\aleph_{\alpha}\right\}$. Clearly $k\{T\}_{\alpha}$ is a subgroup of $k\{T\}$. Let $G$ be a nonzero totally ordered Abelian group. For $a, b \in k\{G\}$ let $(a b)(g)=\sum_{x \in G} a(x) b(g-x)$. It is well known [5] that, under this multiplication, $k\{G\}$ is a totally ordered field. Let $\alpha$ be a nonzero ordinal number; then $k\{G\}_{\alpha}$ is a subfield of $k\{G\}$. Further, $d$ restricted to $k\{G\}_{\alpha}$ is a natural valuation of $k\{G\}_{\alpha}$, its value group being $G$ and its residue class field $k$.

Let $G$ be a totally ordered Abelian divisible group that is an $\eta_{1}$-set of power $\boldsymbol{\aleph}_{1}$ and let $K=R\{G\}$. It was shown in [2] that $K$ is a realclosed field that is an $\eta_{1}$-set and has as its residue class field the reals; thus $K$ might be conjectured to be isomorphic to a residue class field of $C(X)$ for some $X$. However $K / O$ is isomorphic to $R\left\{G^{+}\right\}$which is of power $2^{\aleph_{1}}$, whereas $G$ is of power $\aleph_{1}$; thus, by Theorem $1.3, K$ is not exponentially closed and hence not isomorphic to any residue class field of $C(X)$ for any space $X$.

3. Sufficient conditions. Let $k$ be an Archimedean field, $\alpha$ a nonzero ordinal, $G$ a nonzero totally ordered Abelian group, and let $K=k\{G\}_{\alpha}$. The valuation ideal of $K$ is $k\left\{G^{-}\right\}, G^{-}$being the set of all negative elements of $G$. It has been shown [5] that given a nonzero element $q$ of $P$ then the semigroup $\omega s(q)\left(=\bigcup_{n \in N} n s(q)\right)$ of $G$ is antiwellordered, and further given $g$ in it there exists $m \in N$ such that $g \in \bigcup_{n=1}^{m} n s(q)$. Thus given a sequence $\left(a_{n}\right)_{n \in N}$ in $k, r=\sum_{n=1}^{\infty} a_{n} q^{n}$ is a well defined element of $P$. Further, given $b \in K, r b=\sum_{n=1}^{\infty} a_{n} q^{n} b$.

For $q \in P$ let $\exp q=\sum_{n=0}^{\infty} q^{n} / n$ ! and let $\log 1+q$ $=\sum_{n=1}^{\infty}(-1)^{n-1} q^{n} / n$. By direct calculation it is seen that for all $q, r \in P, \exp q \exp r=\exp q+r$. From analysis we know that $\sum_{n=1}^{\infty}(-1)^{n-1}\left(\sum_{m=1}^{\infty} x^{m} / m !\right)^{n} / n$ converges for all real $x$ such that $|x|<\log 2$; and further that the sum of this series, since it is the ex- 
pansion of $\log e^{x}$, is $x$. Hence the coefficients of this series are the same as the coefficients of the power series $x$. Thus $\log \exp q=q$ for all $q \in P$, proving that exp maps $P$ onto $1+P$ and is one-to-one.

Let $K$ be a non-Archimedean field with value group $G$ and residue class field $k$. We will say that $K$ is properly imbedded in $k\{G\}$ if it is imbedded in $k\{G\}$ such that given $a \in K, V(a)=d(a)$, and such that $k\{G\}_{0} \subset K$. Generalizing somewhat a well known result stated by Conrad [3, p. 328] we get the following: if $K$ is real-closed it can be properly imbedded in $k\{G\}$.

THEOREM 3.1. A non-Archimedean field $K$ with valuation ring $O$, valuation ideal $P$, value group $G$ and residue class field $k$ is exponentially closed if the following hold: (0) $K$ is root-closed, (1) $k$ is exponentially closed, (2) $K / O$ is order isomorphic to $G$, and (3) $K$ may be properly imbedded in $k\{G\}$ in such a way that if $q \in P$ then $\exp q$ and $\log 1+q$ $\in K$.

Proof. Let $K$ be imbedded in $k\{G\}$ such that condition (3) holds; thus exp is an order preserving isomorphism of $P$ onto the multiplicative group of $1+P$. Let $\bar{k}=k 1$. Clearly the ring $O$ is the direct sum of $\bar{k}$ and $P$, the order on the sum being lexicographic. By condition (1), $k$ is exponentially closed; thus given $a \in k, a>1$, the mapping $x \rightarrow a^{x}$ is an exponential in $k$. For $y \in O$ let $y=x+q, x \in \bar{k}$ and $q \in P$, this decomposition being unique. Let $f_{0}(y)=a^{x} \exp q$. Clearly $f_{0}$ is an order preserving isomorphism of $O$ onto the group of positive units of $O$. The additive group of $K$ is the direct sum of $K / O$ and $O$, the order in the sum being lexicographic. An element $u$ in $K$ can be expressed uniquely as $z+y, z \in K / O$ and $y \in O$. By condition (2) there exists an order preserving isomorphism $t$ of $K / O$ onto $G$. Let $f(u)$ $=\left(t(z), f_{0}(y)\right)$. The valuation $V$, restricted to $K^{+}$, is an order preserving homomorphism of the multiplicative group of $K^{+}$(which is divisible by condition (0)) onto $G$ whose kernel is the group of positive units of $O$. Thus the totally ordered group $K^{+}$is the direct product of $G$ and the group of multiplicative units of $O$, the order being lexicographic. Hence $f$ becomes an exponential function of $K$, proving that $K$ is exponentially closed, proving the theorem.

Note. Conditions (0), (1) and (2) are necessary for $K$ to be exponentially closed.

Let $E$ be an $\eta_{1}$-set of power $\aleph_{1}$ and let $\left(x_{n}\right)_{n \in N}$ be a strictly increasing sequence in $E$. Let $E_{n}=\left\{x \in E: x<x_{n}\right\}$. Then $E_{n}$ is an $\eta_{1}$-set of power $\aleph_{1}$. Let $E^{\prime}=\bigcup_{n \in N} E_{n}$. Since $E^{\prime}$ has a countable cofinal sequence it is not an $\eta_{1}$-set. Let $G=R\left\{E^{\prime}\right\}_{1}$ and let $G_{n}=R\left\{E_{n}\right\}_{1}$. Then $G_{n}$ is an Abelian divisible group that is an $\eta_{1}$-set of power $\aleph_{1}$ [2]. Further, 
$G=\cup_{n \in N} G_{n}$; thus $G^{+}$is order isomorphic to $E^{\prime}$ which, under the natural valuation $d$, is the value set of $G$ (cf. Corollary 1.4).

$K=R\{G\}_{1}$ is a real-closed field (hence a root-closed field) that has the reals as its residue class field; thus $K$ satisfies conditions (0) and (1). $K / O$ is isomorphic to $R\left\{G^{+}\right\}_{1}$ which, since $G^{+}$is isomorphic to $E^{\prime}$, is isomorphic to $R\left\{E^{\prime}\right\}_{1}$ : i.e., to $G$; thus $K$ satisfies condition (2). Clearly condition (3) holds. Thus, by Theorem $3.1, K$ is exponentially closed. However, since $K$ has a countable cofinal sequence it is not an $\eta_{1}$-set.

\section{BIBLIOGRAPHY}

1. N. L. Alling, On ordered divisible groups, Trans. Amer. Math Soc. 94 (1960), 498-514.

2. - A characterization of Abelian $\eta_{\alpha}$-groups in terms of their natural valuation, Proc. Nat. Acad. Sci. 47 (1961), 711-713.

3. P. Conrad, On ordered division rings, Proc. Amer. Math. Soc. 5 (1954), 323-328.

4. L. Gillman and M. Jerison, Rings of continuous functions, Van Nostrand, Princeton, N. J., 1960.

5. B. H. Neumann, On ordered division rings, Trans. Amer. Math. Soc. 66 (1949), 202-252.

6. O. F. G. Schilling, The theory of valuations, Math. Surveys, No. 4, Amer. Math. Soc., New York, 1950.

Purdue University 